Conclusions: The disulfide shift of the thiol-disulfide balance and the correlation between the clinical parameters and the thiol-disulfide balance components suggesting the presence of oxidative stress in FMS patients suggests that the role of thiol-disulfide balance in the etiopathogenesis of FMS.

Disclosure of Interest: None declared

DOI: 10.1136/annrheumdis-2018-eular.5754

\section{THU0510 HYPERSENSITIVITY, ALEXITHYMIA AND DISEASE CHARACTERISTICS OF PATIENTS PRESENTING WITH SYMPTOMS OF FIBROMYALGIA}

H. Jacobs ${ }^{1}$, B.A. Grillet ${ }^{2}$, D. De Bacquer ${ }^{3} .{ }^{1}$ Physical Medecine and Rehabilitation, AZ Maria Middelares Ziekenhuis, Ghent, Belgium; ${ }^{2}$ Rheumatology, Reumazorg Zuid West Nederland, Terneuzen, Netherlands; ${ }^{3}$ Departement of Public Health, Ghent University, Ghent, Belgium

Background: Fibromyalgia (FM) is characterised by chronic widespread pain and other symptoms such as fatigue, sleep disturbances and cognitive problems. Psychosocial factors and personality traits may be present in a variable degree and may play a role in the perception, coping and treatment of FM.

Objectives: We looked for symptoms of hyperventilation, depression, anxiety and burn-out as well as for personality traits such as hypersensitivity and alexithymia in a large sample of patients. We looked at differences in prevalence of these symptoms in the population when selected by the 1990 FM classification criteria and by the 2010 diagnostic FM criteria.

Methods: A large group of patients presenting on the Unit of Physical Medicine between 2014 and 2017 with chronic widespread pain was analysed and grouped depending on the 1990 ACR classification criteria for FM on one hand and the 2010 ACR diagnostic criteria for FM on the other hand. Self-administered tools used were the Nijmegen questionnaire (hyperventilation), the Aron questionnaire (hypersensitivity), the HADS questionnaire (anxiety and depression), the BMS-10 questionnaire (burn-out) and the TAS alexithymia questionnaire.

Results: Out of 1085 patients, 828 (76\%) fulfilled the 1990 ACR classification criteria for FM. Hyperventilation (score $\geq 12$ ) was found in $97 \%$ and hypersensitivity (score $\geq 12$ ) in $75 \%$. Symptoms of anxiety and depression (scores $\geq 11$ ) were observed in $59 \%$ and $36 \%$ respectively. Burn-out (score $>4.5$ ) was seen in $40 \%$ of patients while a high TAS score $(\geq 8)$ was found in $70 \%$ of patients. Overall, 906 patients fulfilled the 2010 ACR diagnostic criteria for FM. The distributions of disease characteristics and personality traits in this population were very similar Only 38 out of these 906 patients had a wide spread pain index (WPI) of 3 to 6 .

Conclusions: A significant number of patients demonstrated high levels of anxiety, more than depression. Hypersensitivity and alexithymia were also found to be highly prevalent. There was a substantial level of hyperventilation. It may be useful to consider all these characteristics in the development of treatment programs for FM patients. Results were similar when groups were selected by 1990 ACR classification criteria for FM or by the 2010 ACR diagnostic criteria for FM. Only a minority of patients had a WPI of 3 to 6 .

\section{REFERENCES:}

[1] Van Dixhoorn J, et al. 1985.

[2] Wolfe, et al. 1990.

[3] Aron, et al. 1997.

[4] Maslach, et al. 1996

[5] Wolfe, et al. 2010.

[6] Maes, et al. 2014

Disclosure of Interest: None declared

DOI: 10.1136/annrheumdis-2018-eular.2726

\section{THU0511 EFFECTS OF ADD-ON TRANSCRANIAL DIRECT CURRENT STIMULATION ON PAIN IN KOREAN PATIENTS WITH FIBROMYALGIA}

J.-H. Kang, K.-E. Lee, D.-J. Park, S.-S. Lee. CHONNAM NATIONAL UNIVERSITY MEDICAL SCHOOL AND HOSPITAL, Gwangju, Korea, Republic of Ireland

Objectives: Despite promising preliminary results of transcranial direct current stimulation (tDCS) treatment in patients with fibromyalgia (FM), several issues need to be addressed, including its limited efficacy, low response rate, and poor tolerability. We investigated the efficacy and safety of tDCS as an add-on treatment for chronic widespread pain in Korean patients with FM.

Methods: This study enrolled 38 patients, who were refractory to pain medications, seen at Chonnam National University Hospital from May 2016 to December 2016. A conventional tDCS device was used to supply $2 \mathrm{~mA}$ of current for $20 \mathrm{~min}$ on 5 consecutive days. The anode was placed over the primary motor cortex (M1) and the cathode was located contralateral supraorbital area. The primary end point was a change in visual analogue scale (VAS) pain score at the end of treatment and secondary end points included changes in Fibromyalgia Impact Ques tionnaire (FIQ), Brief Pain Inventory (BPI), Brief Fatigue Inventory (BFI), Beck Depression Inventory (BDI), State-Trait Anxiety Inventory (STAI), and Medical Outcomes Study Sleep Scale scores.

Results: After tDCS, 38 patients showed clinical improvements in the VAS pain score on days 6,13 and 36 compared with day $0(p=<0.001)$. However, improvement of FIQ scores was only seen at day 36 . The BPI and BDI were significantly decreased on days 6 and 13, while BFI and STAI-I were significantly improved only at day 6 . The most of improved indices were not maintained until day 36. There were no significant improvements in Sleep Scale scores after tDCS at days 6,13 , and 36. No serious adverse event was observed.

Conclusions: Our results suggest that tDCS has the potential to produce significant pain relief in FM patients, and may constitute an effective add-on treatment for these patients.

Disclosure of Interest: None declared

DOI: 10.1136/annrheumdis-2018-eular.2738

\section{THU0512 HYPERBARIC OXYGEN THERAPY (HBOT) TREATMENT IN FIBROMYALGIA}

M. Muratore $^{1}$, L. Quarta ${ }^{2}$, P. Sarzi Puttini ${ }^{3}$, C. Cosentino ${ }^{4}$, A. Grimaldi ${ }^{1}$, E. Quarta ${ }^{2}$ ${ }^{1}$ O.U. of Rheumatology, Galateo Hospital, ASL-LE, San Cesario di Lecce; ${ }^{2}$ O.U. of Rheumatology, Galateo Hospital, ASL-LE, San Casario di Lecce; ${ }^{3}$ U.O. Reumatologia- Ospedale "Luigi Sacco", Milano; ${ }^{4}$ Dipartimento di Medicina e Chirurgia, Università di Parma, Parma, Italy

Background: Chronic pain conditions, such as fibromyalgia(FM), are among the most common health problems managed by general practitioners, rheumatologists, clinical psychologists.FM is characterised by multifocal pain, fatigue, non restorative sleep, cognitive complaints high levels of distress, associated with greater affect intensity. There's evidence from randomised controlled trials that some treatments like pharmacotherapy, patient education,behavioural therapy and physiotherapy are effective in reducing symptoms;however the majority of the patients aren't satisfied with the current treatments. HBOT showed some clinical effects that may induce a significant improvement of the FM symptoms.

Objectives: The goal of this work was to evaluate the effect of HBOT on FM symptoms.

Methods: 33 female patients aged 29-63 y, with FM were included in this work. Patients initially pharmacologically treated(Pregabalin $150 \mathrm{mg} / \mathrm{die}$, Duloxetine $60 \mathrm{mg} / \mathrm{die}$ ) with unsatisfactory clinical improvement, were enrolled at the Rheumatology Unit San Cesario-Italy. The HBOT protocol comprised 20 sessions,3d/ $\mathrm{w}, 90 \mathrm{~min}, 100 \%$ oxygen at $2.5 \mathrm{ATA}$. Patients were randomly assigned to treated and control groups and evaluated every month for the next 4 months:patients in group A were treated with 20 sessions of HOBT in the first 2 months and evaluated for the following 2 months; patients in group $B$ used the pharmacological treatment for the first 2 months and then were treated with 20 session of HOBT in the last 2 months. During HOBT no pharmacological treatment was allowed. The treated group patients were evaluated at baseline and after 10 and 20 HBOT sessions. Evaluations consisted of physical examination, including tender point count, and socio-demographic and clinimetric questionnaires:Fibromyalgia Impact Questionnaire(FIQ), Functional Assessment of Chronic IllnessTherapy, Pittsburgh Sleep Quality Index, Quality of life, Beck Depression Inventory, State Trait Anxiety Inventory, Pain Catastrophizing Scale.

Results: 5 patients withdrew from the HBOT treatment for claustrophobia.HBOT led to significant amelioration of all FM symptoms, with significant improvement in life quality.HOBT leads to a reduction in the number of tender points in the 2 groups. This reduction occurs in the group $A$ without changing during the following 2 months of osservation. In the group $B$ the improvement is more related to the HOBT than to the therapy. The FIQ score improves in group A. No improvement was observed in the control group.

Conclusions: The analgesic effects of HBOT have been studied in nociceptive, in inflammatory and neuropathic pain models, and may be useful for the treatment of various chronic pain syndromes. Excessive pain in FM may be due to hyperex citability of the pain processing pathways and under-activity of the pain inhibiting pathways in the brain. It has been shown that HBOT increases cell metabolism, reduces apoptosis, alleviates oxidative stress, increases neurotrophin and nitric oxide levels by enhancing mitochondrial function in neurons and glial cells, it may even promote the neurogenesis of endogenous neural stem cells. HBOT-induced neuroplasticity also leads to the repair of chronically impaired brain functions. Our data confirm the effecicy of HBOT in treating FM. Further studies are required to evaluate the protocol and to understand the duration of the clinical effect.

Disclosure of Interest: None declared

DOI: 10.1136/annrheumdis-2018-eular.6767 